Enhancement of thickness uniformity of thin films grown by pulsed laser deposition

Félix E. Fernández

Department of Physics, University of Puerto Rico, Mayaguez, Puerto Rico 00680-5000

pp. $421-427$

(NASA-CR-197791) ENHANCEMENT OF

THICKNESS UNIFORMITY: OF THIN FILMS

GROWN BY PULSED LASER DEPOSITION

(Puerto Rico Univ.) $B$ p
N95-26081

Unclas

$63 / 27 \quad 0048536$ 


\title{
Enhancement of thickness uniformity of thin films grown by pulsed laser deposition
}

\author{
Félix E. Fernández \\ Department of Physics, University of Puerto Rico, Mayaguez, Puerto Rico 00680-5000
}

(Received 23 September 1994; accepted 19 November 1994)

A peculiarity of the pulsed laser deposition technique of thin-film growth which limits its applicability is the very rapid drop of resulting film thickness as a function of distance from the deposition axis. This is due to the narrow forward peaking of the emission plume characteristic of the laser ablation process. The plume is usually modeled by a $\cos ^{n} \theta$ function with $n$ greater, and in some cases much higher, than 1 . Based on this behavior, a method is presented to substantially enhance coverage uniformity in substrate zones of the order of the target-substrate distance $h$, and to within a specified thickness tolerance. Essentially, target irradiation is caused to form an annular emission source instead of the usual spot. By calculating the resulting thickness profiles, an optimum radius $s$ is found for the annular source, corresponding to a given power in the emission characteristic and a given value of $h$. The radius of this annulus scales with $h$. Calculated numerical results for optimal $s / h$ ratios corresponding to a wide range of values for $n$ are provided for the case of $\pm 1 \%$ tolerance in deviation from the thickness at deposition axis. Manners of producing annular illumination of the target by means of conic optics are presented for the case of a laser beam with radially symmetric profile. The region of uniform coverage at the substrate can be further augmented by extension of the method to multiple concentric annular sources. By using a conic optic of novel design, it is shown also how a single-laser beam can be focused onto a target in the required manner. Applicability of the method would be limited in practice by the available laser power. On the other hand, the effective emitting area can be large, which favors extremely high growth rates, and since growth can occur uniformly over the whole substrate for each laser pulse, single-shot depositions with substantial thicknesses are possible. In addition, the simultaneity of growth over the complete substrate is desirable when monitoring the growth in situ. (O) 1995:American Vacuum, Society.

\section{INTRODUCTION}

Pulsed laser deposition (PLD) is a valuable thin-film growth technique for various classes of technologically important materials. Characteristics of PLD have been reviewed in very comprehensive papers by Cheung and Sankur, ${ }^{1}$ and by Saenger. ${ }^{2,3}$ A peculiarity of PLD is the sharply directional nature of the evaporant plume emitted from the ablation spot at the target. This results in deposition yields which depend strongly on the angle $\theta$ from the target normal, and therefore in deposited film thickness which diminish very rapidly as a function of distance from the substrate center, assumed to be directly opposite the ablation spot. While this can be useful in certain applications calling for very restricted coverage, it can limit the application of PLD in the production environment.

In order to cover larger substrate areas with films of uniform thickness one can resort to rotating the substrate around an axis parallel to, but suitably displaced from, the target normal. Equivalently, one can scan the laser beam over the target, describing a circle with appropriate radius, and centered directly opposite the substrate center. A disadvantage of these approaches is that growth does not occur simultaneously over the substrate. Rather, coverage can be uniform, at best, only each time the substrate (or the beam being scanned) completes one turn. However, since growth is sequential, rather than continuous, along a circle on the substrate, there will be at least some thickness oscillations along this circle. These procedures make film monitoring during growth more difficult, particularly if the substrate is moving. Also, specially for fast rates, the relative importance of lateral growth could lead to higher defect densities.

An alternate approach to improve substrate coverage uniformity with PLD is presented in this work. This consists essentially of illuminating the target with concentric annular zones with approximately the same fluence. Advantages of this approach are that (1) film growth is uniform for any given pulse, (2) thickness oscillations along the azimuthal direction of the substrate will not occur, and (3) for fixed fluence at the target, the total emitting area can be quite large, which implies extremely fast growth rates over large substrate areas. If the angular distribution of the evaporant flux emitted by a small area source is known, then the series of radii of these zones that will yield uniform thickness across a substrate can be calculated. Some exotic resonator designs can yield annular laser outputs which naturally lend themselves to annular illumination. More generally, conic optics can be used to shape a laser beam with radially symmetric intensity distribution into a multiplicity of concentric rings. An example of an optical system including a conic lens (i.e., an axicon) suitable for the case of a Gaussian TEM 00 laser output is presented in Sec. VI.

Small-area thermally equilibrated effusive (i.e., collisionless) sources are known to obey Knudsen's cosine law of emission. ${ }^{4,5}$ For this case, Holland and Steckelmacher ${ }^{6}$ showed that it is possible to obtain very good thickness uniformity for films deposited from thin annular sources. If $h$ is the distance from the source plane to the substrate plane, and 


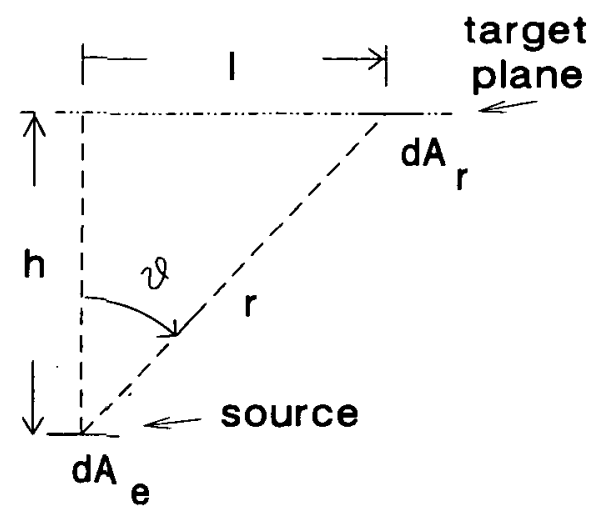

FIC. 1. Target-substrate geometry for small-area source. The target and substrate planes are assumed to be parallel and at a distance $h$ from each other. Substrate surface element $d A_{r}$ is located a distance $l$ from the target center, and a distance $r$ from the source, $d A_{e}$.

$l$ is the radial coordinate in the substrate plane, as shown in Fig. 1, then thickness uniformity within $1 \%$, for example, can in principle be maintained up to values of $l / h$ near 0.5 if the annular source radius is approximately $0.75 h$. Multiple rings have actually been used as evaporation sources in order to obtain films with good thickness uniformity over relatively large areas. ${ }^{7}$

For the case of PLD, the flux generated at the target is usually modeled as a $\cos ^{n} \theta$ function, with $n>1$, often of order 10 , although in some cases much narrower emission cones have been observed, particularly for high ambient pressures during deposition (e.g., hundreds of mTorr). In some cases, the plume has been modeled by the sum of two cosine functions with different powers. The treatment in the following assumes that a single cosine function is sufficient. It would have to be modified accordingly in situations not satisfying this assumption. The forward "beaming" of the flux in PLD is attributed to the effect of collisions, both among the particles forming the plume, and between these and the gas in the deposition chamber. ${ }^{2,8-10}$ In usual practice, the latter type of collisions are relevant only when a gas is purposefully introduced in the chamber.

\section{FILM COVERAGE DISTRIBUTION FOR SMALL- AREA SOURCE}

In this section, an expression for the film thickness, normalized to that at substrate center $\left(d / d_{0}\right)$, is derived assuming a $\cos ^{n} \theta$ flux distribution from the target, where $\theta$ is the angle measured from the target surface normal (the zenith angle). The geometry is shown in Fig. 1, with notation following Glang. ${ }^{5}$ Let $\Gamma$ be the mass evaporation rate per unit area, and $d A_{e}$ be an element of area of source. If $M_{e}$ is the total mass emitted by $d A_{e}$ in a period of time $\tau$, its relationship with $\Gamma$ is given by

$$
M_{e}=\int_{\tau} \int_{A_{e}} \Gamma d A_{e} d t
$$

In general, $\Gamma$ is a function of position in the emitting area and depends on details of the heating process and the thermal diffusivity of the target material. In the following, $\Gamma$ will be replaced by its average over the irradiated target surface, for any given time. Assuming the emission to be independent of the azimuth angle, i.e., a function of $\theta$ only, the requirement that $M_{e}$ be the total mass emitted by the source into a hemisphere leads to the following expression for the mass emitted by this area into a solid angle $d \Omega$ :

$$
d M_{e}=\frac{n+1}{2 \pi} M_{e} \cos ^{n}(\theta) d \Omega .
$$

For the case in which the substrate is parallel to the target plane, the mass received into an arbitrarily located element of substrate area $d A_{r}$ is then

$d M_{r}(\theta)=\frac{n+1}{2 \pi} M_{e} \cos ^{n}(\theta) \frac{d A_{r} \cos \theta}{r^{2}}$,

where $r$ is the distance from element $d A_{e}$ to element $d A_{r}$. Assuming for simplicity unit sticking coefficient, the film thickness at $d A_{r}$ after time $\tau$ is

$$
d=\frac{1}{\rho} \frac{d M_{r}}{d A_{r}}=\frac{1}{\rho} \frac{n+1}{2 \pi} M_{e} \frac{\cos ^{n+1} \theta}{r^{2}},
$$

where $\rho$ is the density of the film material. From the geometry, $\cos \theta=h / r$, and $r^{2}=h^{2}+l^{2}$. Making these substitutions and simplifying in the previous expression, one obtains

$d=d(l)=\frac{n+1}{2 \pi} \frac{M_{e}}{\rho} \frac{1}{h^{2}\left[1+(l / h)^{2}\right]^{(n+3) / 2}}$.

With $d_{0}=d(0)$, the normalized film coverage due to a smallarea source can then be written as

$$
\frac{d}{d_{0}}=\left[1+(l / h)^{2}\right]^{-(n+3) / 2}
$$

\section{FILM COVERAGE DISTRIBUTION FOR THIN ANNULAR SOURCES}

\section{A. Single annular source}

Suppose now that the laser light is focused onto the target in a thin annulus of radius $s$ and width $\Delta s$, and with no change in irradiance along the circumference. The substrate is again assumed to be parallel to the target surface. The geometry is shown in Fig. 2. The distances $h, l$, and $r$ are as defined before.

The thickness of the material received at an element of area in the substrate, again assuming unit sticking coefficient, must be calculated now taking into account contributions from the complete ring. Using the result in Eq. (4) and definition (1), the total thickness at $d A_{r}$ can be evaluated from

$$
d=\frac{n+1}{2 \pi \rho} \int_{\tau} \int_{s} \int_{\alpha} d t d A_{e} \frac{\Gamma \cos ^{n+1}(\theta)}{r^{2}},
$$

where

$$
d A_{e}=s d s d \alpha,
$$

and $\alpha$ is the angle, measured on the substrate plane, between the line $l$ from the geometry axis to $d A_{r}$ and the projection onto the substrate plane of the radius line $s$ from the ring 


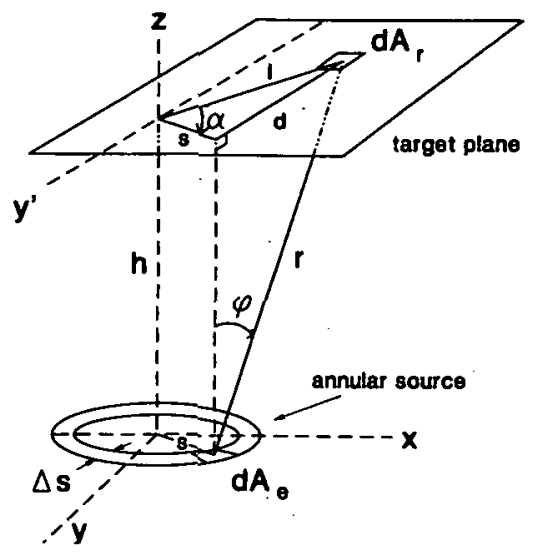

FIG. 2. Target-substrate geometry for thin annular source with radius $s$. Angle $\alpha$ is that between the line $(l)$ from substrate center to element $d A_{r}$ and the projection onto the substrate plane of the radius line to source element $d A_{e}$. Angle $\phi$ is measured between the normal to the source element $d A_{e}$ and the line $(r)$ between $d A_{e}$ and the receiving element $d A_{r}$. Notation follows Ref. 5.

source center up to $d A_{e}$. In this integral $\alpha$ sweeps one full circle. If the ring is considered thin (i.e., its width much smaller than its radius), the integral over $s$ can be eliminated; then Eq. (1) is simply

$$
M_{e}=2 \pi s d s \int_{\tau} d t \Gamma .
$$

Recalling that $\cos \theta=h / r$, Eq. (7) can be written as

$$
d=\frac{n+1}{(2 \pi)^{2} \rho} M_{e} \int_{\alpha} d \alpha \frac{h^{n+1}}{r^{n+3}} .
$$

From the configuration geometry, $r$ can be expanded in terms of the other parameters and variables:

$$
r^{2}=h^{2}+l^{2}+s^{2}-2 s l \cos \alpha .
$$

Substituting this in Eq. (10), one obtains

$$
\begin{aligned}
d= & \frac{(n+1) M_{e}}{(2 \pi)^{2} \rho h^{2}} \int_{0}^{2 \pi} d \alpha\left[1+(l / h)^{2}+(s / h)^{2}\right. \\
& \left.-\frac{2 s l}{h^{2}} \cos \alpha\right]^{-(n+3) / 2} .
\end{aligned}
$$

At $l=0$ this can be integrated easily to give

$$
d_{0}=\frac{(n+1) M_{e}}{2 \pi \rho h^{2}} \frac{1}{\left[1+(s / h)^{2}\right]^{(n+3) / 2}} .
$$

Combining Eqs. (12) and (13) one finally obtains the expression for the normalized film thickness due to an annular source,

$$
\begin{gathered}
\frac{d}{d_{0}}=\frac{1}{2 \pi}\left[1+(s / h)^{2}\right]^{(n+3) / 2} \int_{0}^{2 \pi} d \alpha\left[1+\left(\frac{l}{h}\right)^{2}\right. \\
\left.+\left(\frac{s}{h}\right)^{2}-\frac{2 s l}{h^{2}} \cos \alpha\right]^{-(n+3) / 2} .
\end{gathered}
$$

\section{B. Multiple concentric annular sources}

The result in Eq. (14) can be generalized for the case of a multiplicity of concentric thin annular sources. The simplest situation, but also the one of greater practical interest, is obtained when all illuminated regions at the target receive the same intensity. Then the elemental emission distribution can be described, at least approximately, by the same $\cos ^{n} \theta$ function. Further, the mass evaporation rate $\Gamma$ can then be assumed to be the same for all illuminated areas. The total mass emitted by the $i$ th ring, with radius $s_{i}$ is

$$
M_{e i}=2 \pi s_{i} d s \int_{t} \Gamma d t
$$

For simplicity, the (small) width of all the rings is now assumed to be the same $(d s)$. Relaxation of this condition leads only to straightforward modification in the following results.

The total thickness at any given element of area $d A_{r}$ of the substrate can now be calculated by adding the contributions of each of the rings. The individual contribution of each ring source is calculated by using Eq. (12), with evident replacements. As was done before, the total thickness at substrate center $(l=0)$ can be evaluated, and this can be used to find the final expression for the normalized thickness as a function of radial distance $l$ from the substrate center. For $N$ ring sources with radii $s_{1}, s_{2}, \ldots, s_{N}$, this is,

$$
\frac{d}{d_{0}}=\frac{\sum_{i=0}^{N} s_{i} \int_{0}^{2 \pi} d \alpha\left[1+\left(\frac{l}{h}\right)^{2}+\left(\frac{s_{i}}{h}\right)^{2}-\frac{2 s_{i} l}{h^{2}} \cos \alpha\right]^{-(n+3) / 2}}{\sum_{i=1}^{N} s_{i}\left[1+\left(s_{i} / h\right)^{2}\right]^{-(n+3) / 2}} .
$$

Once the exponent $n$ describing the small-source distribution is experimentally determined for a given material under specified conditions, expression (16) [or (14), for a single ring] can be used to model substrate coverage in terms of the $s_{i}$ parameters. If annular sources with these radii and the same emission characteristics can be produced, then coverage approaching the model results should be possible. In the following section examples for particular cases are given; then, in Sec. VI, a manner of producing annular patterns on the target is presented.

\section{OPTIMIZATION EXAMPLES}

Prior to determination of optimum annular source radii for any given geometry, the small area emission profile for the target material of interest must be known. "Small area" means that the spot diameter is small compared to the targetsubstrate distance. To a greater or lesser degree, the profile depends on factors such as fluence, power density, laser wavelength, spot size, gas pressure, and target condition. In going over to a configuration in which the target is illuminated by one or more concentric rings, these factors must be controlled so that the small-area profile remains the same and the model results can be borne out. The previous treatment assumes that the profile can be described by a $\cos ^{n} \theta$ function. Result (6) can be used to fit thickness measurements in order to determine $n$. 


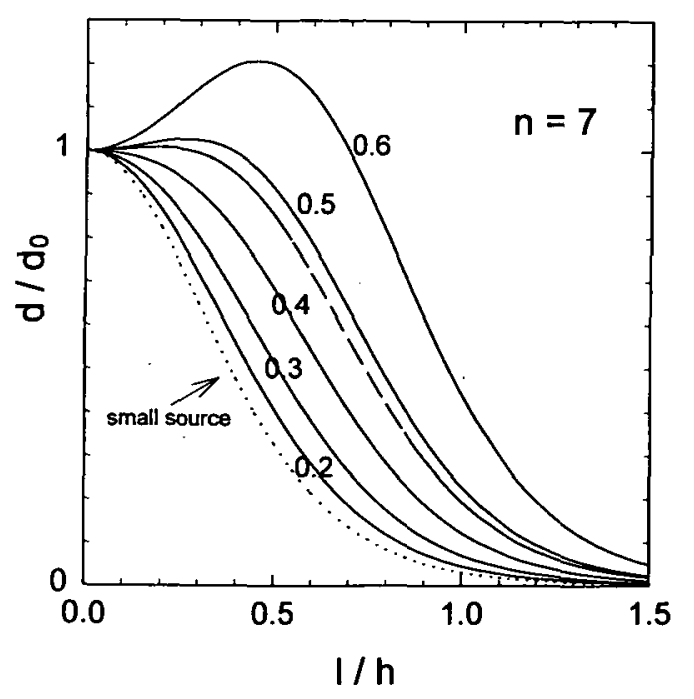

Fig. 3. Thickness profile, normalized to central thickness, as a function of substrate radial parameter $l / h$ for target emission distribution of form $\cos ^{7} \theta$ and small source $(\cdots)$, thin annular sources with increasing radii, $s / h=0.2$, $0.3,0.4,0.5,0.6(-)$, optimum ratio for $\pm 1 \%$ tolerance variation, $s / h$ $=0.475(---)$.

It is assumed now for illustrative purposes that $\cos ^{n} \theta$, with $n=7$ has been determined to fit experimental results at a fixed fluence for a given material irradiated in a small area. The radius $s$ of a single thin annular source producing the most uniform coverage in the central region of the target can be determined by using Eq. (14) to calculate the normalized thickness as a function of $l / h$, with $s / h$ as a parameter. For the case $n=7$, results of calculations for a range of $s / h$ values $(0.2-0.6)$ are presented in Fig. 3 with solid lines. For comparison, the profile due to a small source is shown in the same figure with a dotted line. By adjusting the $s / h$ value correctly, a distribution can be obtained which would be flat, within a specified tolerance, up to a certain radial boundary. The latter is smaller for tighter tolerances of coverage uniformity. The dashed curve in Fig. 3 gives the result for $s / h$ $=0.475$, which would yield flat coverage within a $\pm 1 \%$ margin. This region extends to $l / h=0.33$ in this case. This means that, for example, if the target-substrate distance is 5 $\mathrm{cm}$, the source radius should be $-2.4 \mathrm{~cm}$, and would yield very uniform coverage for substrates up to $3.3 \mathrm{~cm}$ in diameter. Coverage decays very rapidly outside this region.

Calculated $s / h$ values for flat coverage within a $\pm 1 \%$ band for a range of values of the exponent $n$ in the plume distribution are shown in Table I and Fig. 4. In Table I the maximum radial parameter value $l / h$ for flat coverage is also given. For the restricted $n$ range from 2 to 10 , the optimum $s / h$ value can be represented to a good approximation by the function $0.35+0.5 \exp (-n / 5)$.

One should recall that these results are valid in the limit of thin annular sources. For a fixed width $\Delta s$ of the source, this approximation is therefore worse for geometries with short target-substrate distances. However, as these results scale with $h$, it is clear that, in principle, very large areas can be covered uniformly. The main restriction is set by the available laser power. Assuming the width of the annular
TABLE I. Calculated optimum values, for uniform coverage to within $\pm 1 \%$, of the ratio $(s / h)$ of radius $(s)$ of a thin annular source to the targetsubstrate distance $(h)$, as a function of the exponent $(n)$ in the $\cos ^{n} \theta$ model for plume profile. Maximum extent of uniform coverage in each case is given in terms of the ratio $l / h$, where $l$ is the radial position in the substrate, measured from the deposition axis.

\begin{tabular}{rcc}
\hline$n$ & $s / h$ & $\begin{array}{c}l / h \max . \\
\left( \pm 1 \% d_{0}\right)\end{array}$ \\
\hline 1 & 0.778 & 0.56 \\
2 & 0.690 & 0.48 \\
3 & 0.626 & 0.44 \\
4 & 0.577 & 0.40 \\
5 & 0.538 & 0.37 \\
6 & 0.506 & 0.35 \\
7 & 0.479 & 0.33 \\
8 & 0.456 & 0.31 \\
9 & 0.436 & 0.30 \\
10 & 0.418 & 0.28 \\
15 & 0.354 & 0.24 \\
20 & 0.312 & 0.21 \\
25 & 0.282 & 0.19 \\
30 & 0.260 & 0.18 \\
40 & 0.227 & 0.15 \\
50 & 0.205 & 0.14 \\
100 & 0.146 & 0.09 \\
200 & 0.104 & 0.07 \\
\hline \hline
\end{tabular}

source is made equal to the diameter (a few millimeters) of the small area source commonly employed in PLD, which indeed need not be the case, the surface irradiated increases by a factor of order $2 \pi s$ for each annular source of radius $s$. The energy per pulse required from the laser then increases by this same factor. For the example considered above, with $s=2.4 \mathrm{~cm}$, this means a factor of 150 . Assuming a circular spot $1 \mathrm{~mm}$ in diameter, neglecting losses in the beam delivery, and for $10 \mathrm{~ns}$ pulses, a power density of $10^{8} \mathrm{~W} / \mathrm{cm}^{2}$

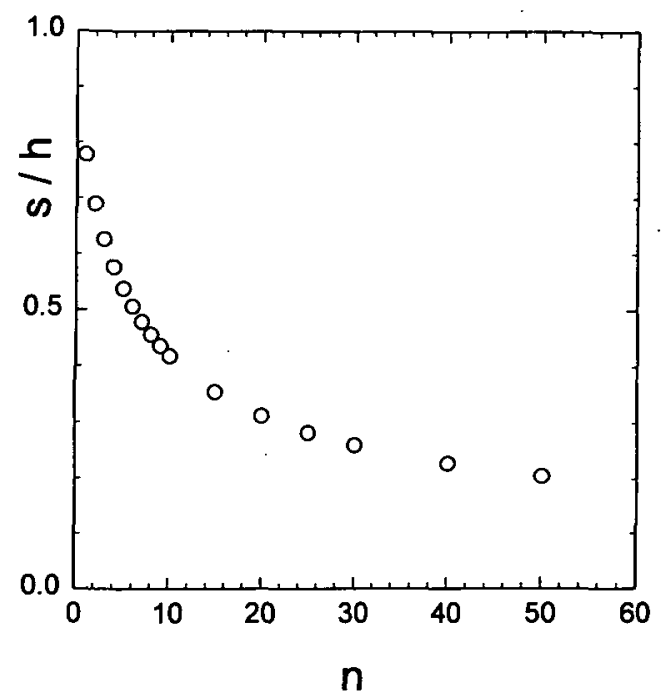

FIG. 4. Best $s / h$ ratios for annular sources as a function of the power $(n)$ in the small source profile distribution. The parameter $s / h$, in each case, indicates the combinations of target-substrate distance $(h)$ and annular source radius $(s)$ which would yield flat coverage within a $\pm 1 \%$ tolerance band within a maximum extent of the substrate. 


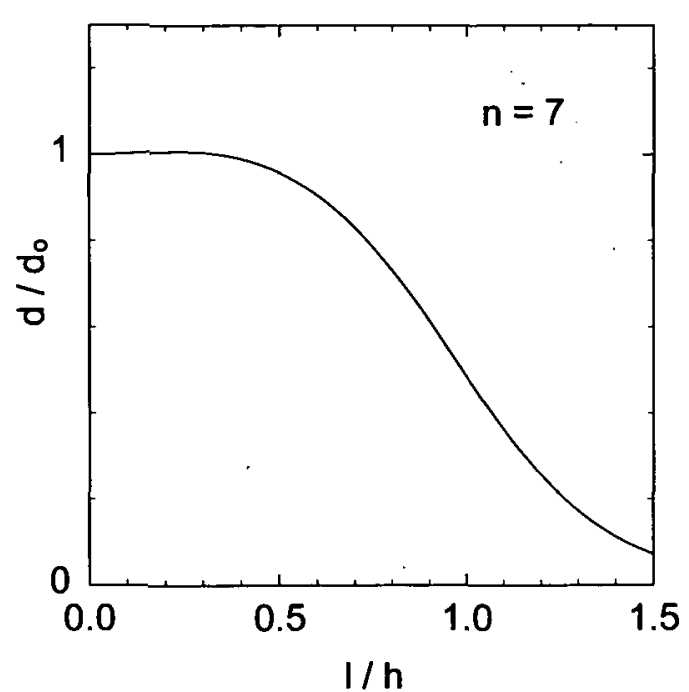

Fig. 5. Calculated normalized thickness profile for two thin concentric annular sources with $s / h=0.38$ and $s / h=0.8$, assuming again a $\cos ^{7} \theta$ small source distribution. The resulting profile is flat to within $\pm 1 \%$ up to $l / h$ $=0.4$.

requires $7.5 \mathrm{~mJ}$ pulses. Thus, an energy of about $1.13 \mathrm{~J}$ per pulse would be required for the annular source of the example. Clearly, the geometry can be tailored in order to make maximum utilization of a high-power laser. Because of the relatively fast drop of coverage after the flat region, and the increase in volume ablated from the target, compared to a small source, very fast deposition rates are possible, including one-shot depositions of relatively thick films.

While the zone of uniform coverage can be enlarged by increasing the target-substrate distance $h$ and the ring source radius, characteristics of the resulting film can depend, in some cases, on the absolute value of $h$. It might then be desirable to keep $h$ fixed. The zone of uniform coverage can still be increased then by using additional, concentric, annular sources. For the same conditions of the previous numerical example, one obtains uniformity within $\pm 1 \%$ up to $l / h$ $=0.4$ by assuming two sources with radial parameters $(s / h)$ 0.38 and 0.8 . The calculated curve for this case is shown in Fig. 5. For the geometry with $h=5 \mathrm{~cm}$, this means $s_{1}=1.9$ $\mathrm{cm}, s_{2}=4.0 \mathrm{~cm}$, and uniform coverage for substrates up to 4 $\mathrm{cm}$ diameter. Again with the same assumptions as before in terms of spot size and power density, energies of $\sim 2.8$ $\mathrm{J} /$ pulse would be required. This is about double the energy requirement that would be required to achieve the same result by adjusting $h$ (and $s$ ) if using a single-ring configuration. Also, more material would be wasted with the two-ring configuration. It should be mentioned that the use of small spot sizes in PLD can produce wider plume distributions. ${ }^{10}$ Thus, annular sources with widths less than about $1 \mathrm{~mm}$ could lead to better coverage uniformity while not placing extraordinary demands on laser pulse energy.

\section{AXICON SEGMENTING FOR A GAUSSIAN BEAM}

Annular output is possible with some types of laser resonators. ${ }^{11}$ These lend themselves naturally to irradiate a

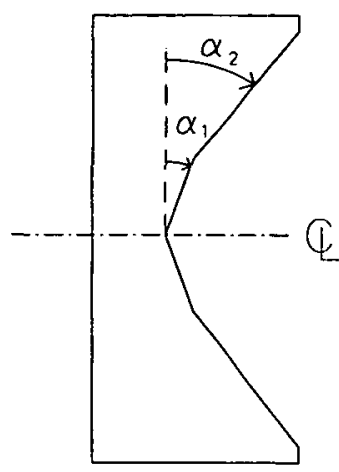

FIG. 6. Negative axicon lens with a two-sheet surface. Each sheet consists of a segment of a cone with apex angle $\alpha_{1}$ or $\alpha_{2}$. The cones are coaxial. The angles would in practice be small in order to prevent excessive losses due to reflection.

target so that a ring source of evaporant is produced. However, other axially symmetric modes are much more common. It is possible in the latter cases to design relatively simple optical systems which will generate concentric ring sources with specified radii. If a single ring source is desired, the optics can consist of an axicon and a regular spherical lens, as shown in Sec. VI. If more than one ring source is required to enhance coverage it is possible, to design a multiple-angle axicon which, in combination with a spherical lens, will meet this requirement. One of the axicon faces is segmented into a number of coaxial conic surfaces with different apex angles. A longitudinal cross section of an axicon with two apex angles is shown in Fig. 6. The angle for each cone must be determined for a specific geometry. This takes into account the required ring source radii, the power of the focusing lens, and the distance between the latter and the target. It should normally be possible to select small values for the axicon angles, which is desirable in order to keep reflection losses in the lens at a minimum. On the other hand, the extent of each of the axicon surfaces is determined by the laser beam intensity distribution. For a "top hat" distribution the situation is quite simple. In the following, the case of a Gaussian $\mathrm{TEM}_{00}$ beam is treated.

For a Gaussian $\mathrm{TEM}_{00}$ laser beam, the intensity distribution (in units of, for example, $W / \mathrm{cm}^{2}$ ) in a plane perpendicular to the beam axis can be written as

$$
I(r)=I_{0} e^{-2 r^{2} / w^{2}},
$$

where, since there is no risk of confusion with its use in the previous sections, now $r$ is the radial coordinate from the beam axis. The parameter $2 w$ is the "spot size" corresponding to a given particular plane. Due to diffraction effects, $w$ is a function of position $(z)$ along the beam axis, and is least at the so-called "beam waist." For lasers with substantial resonator lengths and apertures, such as those suitable for PLD, $w(z)$ is a very slow function. For the case of interest here $2 w$ is assumed to be constant, and equal to the width of the laser beam at the laser output window.

The power $P_{n}$ transmitted through an annular region, concentric with and perpendicular to the beam axis, and bounded by radii $r_{n-1}$ and $r_{n}$ is 


$$
P_{n}=\int_{0}^{2 \pi} \int_{r_{n-1}}^{r_{n}} I(r) r d r d \theta .
$$

Let $r_{0}=0$. Substituting in Eq. (18) the expression (17) for $I(r)$, one obtains

$$
P_{n}=\frac{\pi}{2} I_{0} w^{2}\left[e^{-2\left(r_{n-1} / w\right)^{2}}-e^{-2\left(r_{n} / w\right)^{2}}\right]
$$

The full power in the beam cross section is

$$
P_{\text {total }}=\frac{\pi}{2} I_{0} w^{2}
$$

Now the average power density at each of the annular illuminated regions of the target will be constrained to be the same. Let the radii of these regions be $s_{1}, s_{2}, \ldots s_{N}$. The width of each region is small compared to $s_{1}$. Again, for simplicity, all widths are assumed to be the same $(\Delta s)$. The area of the $n$th annular region in the target is

$$
A_{n}=2 \pi s_{n} \Delta s \text {. }
$$

The requirement above is

$$
\frac{P_{1}}{A_{1}}=\frac{P_{2}}{A_{2}}=\cdots=\frac{P_{N}}{A_{N}}=\text { constant. }
$$

The total power incident on the target is

$$
P_{\text {total }}=\sum_{n} P_{n}
$$

Relations (21)-(23) lead to the following condition for the power transmitted through the $n$th annular zone:

$$
P_{n}=P_{\text {total }} \frac{s_{n}}{\Sigma_{i} s_{i}} .
$$

Using results (19), (20), and (24), one obtains, for the $n$th annular zone in the target (with radius $s_{n}$ ), corresponding to the $n$th annular zone in the axicon (bounded by radii $r_{n-1}$ and $r_{n}$ ),

$$
P_{n}=P_{\text {total }}\left[e^{-2\left(r_{n-1} / w\right)^{2}}-e^{-2\left(r_{n} / w\right)^{2}}\right] \text {. }
$$

Solving for $r_{n} / w$, a recursive formula for the radii $r_{n}$ which defines the zones of the axicon is obtained:

$$
\frac{r_{n}}{w}=\sqrt{\ln \left[e^{-2\left(r_{n-1} / w\right)^{2}}-\left(s_{n} / \Sigma_{i} s_{i}\right)\right]^{-1 / 2}}
$$

The recursion starts with $r_{0}=0$. The required aperture of the axicon lens can be estimated remembering that $99 \%$ of the power in a Gaussian $\mathrm{TEM}_{00}$ beam is contained within a circle with diameter $\pi w$. As an example, for the two-ring source considered at the end of the previous section, with $s_{1}=1.9 \mathrm{~cm}$ and $s_{2}=4.0 \mathrm{~cm}$, formula (26) leads to a radius $r_{1}=0.441 w$ for the inner section of the axicon.

\section{CONFIGURATIONS FOR IMPLEMENTATION}

An ideal configuration for using an axicon in order to produce annular sources in PLD is shown in Fig. 7. This, however, requires the substrate to be placed between the focusing lens $L$ and the target $T$, right after the chamber win-

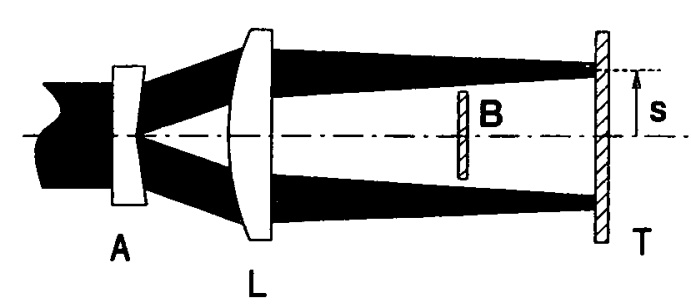

FIG. 7. Configuration for head-on illumination of the target. The negative axicon $A$ shapes the incident laser beam into a cone which is focused by a converging spherical lens $L$ into a ring of radius $s$ at the target $T$. The substrate $B$ is placed facing the target, but in the volume not crossed by the incident light. For single-shot depositions a transparent substrate could extend beyond this region.

dow, which is not shown in the figure. While perhaps not to, convenient for current PLD chamber arrangements, it is certainly possible to design systems allowing this straight-line arrangement. The optical end geometric parameters-axicon angle $\alpha$, focal length of lens $L$, and the distance between these two lenses-can be chosen so that the radius of the conic beam at the chamber window is substantially larger than the desired source radius $s$. Even then, a considerable amount of emitted material can be ejected in the direction of the chamber window, which would have to be protected by a removable glass sheet. Besides simplicity, an advantage of the configuration in Fig. 7 is the fact that it can be chosen so that the beam incides onto the target at a small angle. Some of the problems associated with crater formation when the beam incides at $\sim 45^{\circ}$, i.e., changes in yield and plume orientation, would be minimized. It should be noted also that it is possible to use the head-on geometry to advantage when single-pulse depositions on transparent substrates are required. In this case beam could traverse the substrate, which would in turn protect the window.

In the usual configuration for PLD the target is angled with respect to the laser beam and the substrate is placed facing the target. In Fig. 8 an arrangement using an axicon, but with an inclined target, is presented. In this case the amount of material emitted in the direction of the window is

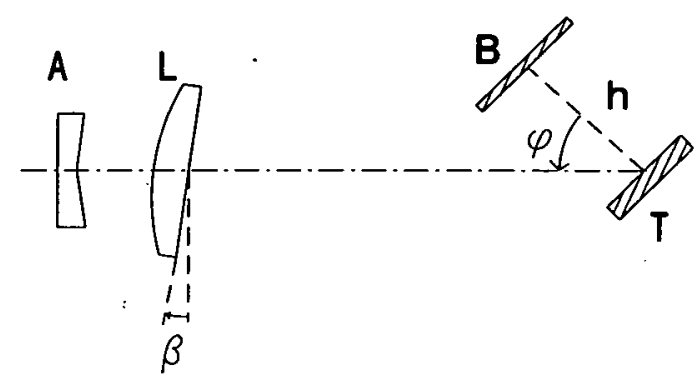

Fig. 8. Configuration for oblique illumination of the target. The target $T$ is inclined so that its normal makes an angle $\phi$ with respect to the laser beam axis. The substrate $B$ is out of the way, facing $T$. In order to produce a circular footprint of the beam on the target surface, lens $L$ is tilted by an angle $\beta$. 
much less. In addition, the window aperture can be smaller. On the other hand, inclination of the target introduces two difficulties. First, the beam footprint on the target will be elliptic instead of circular. Second, the width of the beam intercepted by the target will not be uniform, and therefore the fluence will not be the same in all illuminated regions of the target. These difficulties can be partially solved by tilting the lens $L$, as shown in Fig. 8. In this manner, the beam footprint can be made circular again. Appropriate tilt angles depend on details of the particular configuration, but can be found easily with a raytrace procedure. Since the optimum tilt has some dependence on the ray height at the focusing lens, the beam width at the target is only partly corrected by this tilt. A few rules of thumb can be gleaned from simple calculations assuming a thin focusing lens. Naturally, the target inclination ( $\phi$ in Fig. 8) should not be too large. The smaller the value of $\phi$ selected, the smaller the tilt angle required. For maximum extent of uniform coverage at the substrate, the bounds of the substrate (or substrate holder), in conjunction with the target-substrate distance $h$ and the source radius $s$, determine the lowest value acceptable for $\phi$, before part of the incident light cone is intercepted by the substrate. Simple geometry leads to an estimate of $\tan ^{-1}[(s$ $+R) / h$ ] for lowest allowable value of $\phi$, where $R$ is the substrate radius. The tilt angle for the focusing lens $L$ which will make the footprint circular again is a very sensitive function of the beam angle at this lens. It is convenient to keep this beam angle as small as possible. It can in fact be made equal to zero by inserting a second axicon before lens $L$ in Fig. 8, similar to the first one, but positive and with larger aperture. Its conic surface would face, and be parallel to, that of the first axicon. Use of this axicon pair would have the effect of making the laser beam parallel to the optical axis again, so that it would incide onto lens $L$ as a hollow cylinder instead of a hollow cone. Introduction of the second axicon would permit additional freedom in the design, even for the head-on configuration, but the requirements for the mechanical alignment of the complete beam delivery optics would be more stringent. For the inclined configuration, more nearly uniform fluence can be obtained by means of longer focal lengths for lens $L$ and shorter defocus lengths. In order to obtain specified source radius and fluence, however, the latter can require larger incident beam heights at lens $L$ (and therefore larger apertures), or nonzero incidence angles for the rays at $L$ (and possibly larger tilt angles required for correction).

Clearly, the head-on configuration is the better alternative. In any case, successful implementation of these schemes ultimately depends on (1) accurate knowledge of the small- source emission distribution characteristic for the conditions at hand, and (2) availability of a pulsed laser source with radially symmetric output of sufficient power. The presence of persistent "hot spots" in the beam profile would render the source useless for the task considered here.

\section{CONCLUSION}

A method has been presented to enhance coverage uniformity in pulsed laser deposition by means of one or more annular emission sources instead of a spot source. Model calculations assuming a $\cos ^{n} \theta$ dependence for the plume distribution show that, in principle, film flatness can be maintained for substrate zones of the order of the target-substrate distance $h$. Calculated numerical results for optimal geometries, in terms of the radius of a single thin annular source and the distance $h$ were given for a wide range of values for $n$. For the case of a laser beam with radially symmetric profile, means of producing the required illumination of the target were proposed by means of conic optics. A novel conic optic for producing multiple annular illumination was described. System configurations which could be employed to take advantage of this approach were suggested. While applicability of the method presented would be limited in practice by the available laser power, it offers considerable advantages over the alternatives of scanning the laser beam or rotating the substrate. In particular, extremely high growth rates are possible, and since growth occurs simultaneously over the whole substrate for each laser pulse, single-shot depositions with substantial thicknesses are viable.

\section{ACKNOWLEDGMENTS}

The author is pleased to recognize the support offered by the U.S. Army Research Office under Grant No. DAAL0390-G-0213, by NASA under Grant No. NAG-5-2188, and by the Puerto Rico NSF-EPSCoR Program through Grant No. EHR-9108775.

1J. T. Cheung and H. Sankur, CRC Crit. Rev. Solid State Mater. Sci. 15, 63 (1988).

${ }^{2}$ K. L. Saenger, Proc. Adv. Mater. 2, 1 (1993).

${ }^{3}$ K. L. Saenger, Proc. Adv. Mater. 3, 63 (1993).

${ }^{4}$ M. Knudsen, Ann. Phys. (Leipzig) 28, 999 (1909).

${ }^{5}$. Glang, in Handbook of Thin Film Technology, edited by L. I. Maissel and R. Glang (McGraw-Hill, New York, 1970), Chap. 1.

${ }^{6}$ L. Holland and W. Steckelmacher, Vacuum 2, 346 (1952).

${ }^{7}$ K. H. Behmt and R. A. Jones, Vacuum 11, 129 (1961).

${ }^{8}$ R. Kelly and R. W. Dreyfus, Nucl. Instrum. Methods B 32, 341 (1988).

${ }^{9}$ R. Kelly, J. Chem. Phys. 92, 5047 (1990).

${ }^{10}$ K. L. Saenger, J. Appl. Phys. 70, 5629 (1991).

${ }^{11}$ A. E. Siegman, Lasers (University Science, Mill Valley, CA, 1986), p. 908 
LANCASTER PRESS, INC., LANCASTER, PA 